\title{
Chromosomal Polymorphism of Two Pickling Cucumbers (Cucumis sativus L.) Revealed by Fluorescent Staining with CMA and DAPI
}

\author{
Yoshikazu Hoshi, ${ }^{1, *}$, Masahiro Mori ${ }^{1}$, Hideyuki Matoba ${ }^{2}$, Norikazu \\ Tagashira $^{3}$, Tatsuro Murata ${ }^{1}$, Wojciech Plader ${ }^{4}$ and Stefan Malepszy ${ }^{4}$ \\ ${ }^{1}$ Department of Plant Science, Tokai University, Kawayo, Minamiaso-mura, \\ Kumamoto, 869-1404, Japan \\ ${ }^{2}$ Department of Applied Biological Science, College of Bioresource Sciences, Nihon University, \\ Kameino 1866, Fujisawa, Kanagawa 252-8510, Japan \\ ${ }^{3}$ Department of Living Design and Information Science, Faculty of Human Development, \\ Hiroshima Jogakuin University, 4-13-1 Ushita Higashi, Higashi-ku, Hiroshima 732-0063, Japan \\ ${ }^{4}$ Department of Plant Genetics, Breeding and Biotechnology, Warsaw Agricultural University, \\ ul Nowoursynowska 166, 02-787 Warsaw, Poland
}

Received September 18, 2007; accepted January 16, 2008

\begin{abstract}
Summary To resolve the discrepancy among previous karyotype descriptions, chromosome comparative study between two closely related cucumber cultivars, C. sativus L. cv. Borszczagowski and cv. Monastyrski, was performed by chromosome preparation technique with enzymatic maceration, and fluorescent banding method with chromomycin $\mathrm{A}_{3}$ (CMA) and 4',6-diamidino-2-phenylindole (DAPI). Heterochromatic band polymorphisms were detected in chromosomes 2, 3, 5 and 7, whereas no chromosomal polymorphism was found in chromosomes 1, 4 and 6. In cv. Borszczagowski chromosome $2, \mathrm{CMA}^{+} \mathrm{DAPI}^{+}$heterochromatic band at terminal region of the long arm was smaller than that of cv. Monastyrski. Chromosome 3 of cv. Monastyrski was characterized by $\mathrm{CMA}^{+} \mathrm{DAPI}^{+}$ bands on the terminal regions of both arms, whereas that of cv. Borszczagowski displayed only on the terminal region of long arm. Distinct $\mathrm{CMA}^{+} \mathrm{DAPI}^{+}$band located at terminal region of the long arm in cv. Borszczagowski was larger than that of $\mathrm{cv}$. Monastyrski. In chromosome 7, terminal $\mathrm{CMA}^{+} \mathrm{DAPI}^{+}$band at the short arm was only observed in cv. Monastyrski. This result suggested that inconsistency of karyotypes among previous reports was due to chromosomal polymorphism.
\end{abstract}

Key words Cucumis sativus L., Chromosomal polymorphism, CMA, DAPI

Cucumis sativus L. (Cucumber, $2 n=2 x=14$ ) is one of the most important crops and is widely cultivated all over the world (Jeffrey 1980). Since the first C-banding on the somatic chromosome was reported by Ramachandran and Seshadri (1986), karyomorphological studies for chromosomal identification have been going on with this species. Recently, several cytogenetists have attempted to characterize the C. sativus chromosomes using basic staining methods (Hoshi et al. 1998, Chen et al. 1998, Koo et al. 2002) and differential staining methods (Ramachandran and Seshadri 1986, Plader et al. 1998, Hoshi et al. 1998, 1999, Chen et al. 1998, Koo et al. 2002, 2005).

Ramachandran and Seshadri (1986) reported chromosome identification at mitotic metaphase and meiotic pachytene stage to compare C. sativus with C. melo. In contrast, Hoshi et al. (1998) and Plader et al. (1998) carried out the chromosome identification with new banding pattern using the improved banding method (Hoshi et al. 1998) and fluorescent differential staining with basespecific fluorescent dyes (Plader et al. 1998). Using these banding patterns, they rearranged chromosomes 1 to 7. On the other hand, Chen et al. (1998) applied C-banding technique for chromosome identification using a Chinese cultivar, and showed the karyotype which was different from

* Corresponding author, e-mail: yhoshi@agri.u-tokai.ac.jp 
those of previous reports. The latest report of the C-banding pattern was done by Koo et al. (2002, 2005), who used a Korean cucumber cultivar and displayed quite different pattern in the haploid karyotype ideogram. Although the differences of these karyotype information were considered due to differences of cultivar materials, chromosome preparation techniques, or growing conditions (Hoshi et al. 1998, Koo et al. 2002), an evidentially experimental investigation to advance the consideration has not been performed. In addition to this, employing the same chromosome preparation technique and banding method among different $C$. sativus cultivars are quite necessary to resolve the discrepancy among the karyotype descriptions, even though each previous banding information was enabled to characterize the own karyotype components.

The intraspecific polymorphism of chromosome or chromosome segment among different cultivars has been demonstrated in several plants such as Secale cereale (Weimarck 1975), Hordeum vulgare (Vosa 1976, Laursen 1978, Kakeda et al. 1991), Scilla sibirica (Vosa 1973a), Leopoldia comosa (Bentzer and Landström 1975), Tulbaghia leucantha (Vosa 1973b) and so on. These plants showed polymorphisms of constitutive heterochromatin revealed by $\mathrm{C}-$, $\mathrm{N}$ - or fluorescent banding method. Moreover, base-specific fluorescent staining methods with chromomycin $\mathrm{A}_{3}$ (CMA) and 4',6-diamidino-2-phenylindole (DAPI) have been commonly employed to localize some GC- and AT-rich heterochromatic regions on chromosome, respectively (Schweizer 1976). Plader et al. (1998) reported that all of $C$. sativus chromosomes were possible to easily identify based on those heterochromatic segments by the improved method for a simplified fluorescent staining. They demonstrated that the fluorescent differential staining with these base-specific fluorochromes allowed stable detection of the heterochromatic bands on chromosomes and rapid identification of each homologous chromosome pair in C. sativus.

In this study, we carried out comparative study of chromosomes between two European pickling type cucumber cultivars by means of fluorescent differential staining, and discussed chromosomal polymorphism.

\section{Materials and methods}

\section{Plant material}

Two pickling type cucumber cultivars, Cucumis sativus L. cv. Borszczagowski and C. sativus cv. Monastyrski were used in this study. These lines were maintained at the Department of Plant Genetics, Breeding and Biotechnology, Warsaw Agricultural University and Applied Plant Science, Tokai University.

\section{Chromosome preparation}

Mitotic chromosome slides were prepared using the enzymatic digestion method. Root tips for the chromosome preparation were collected from two day-old seedlings. The root tips were treated with $0.05 \%$ colchicine at $18^{\circ} \mathrm{C}$ for $2 \mathrm{~h}$ and fixed in $3: 1$ ethanol-acetic acid for $1 \mathrm{~h}$ and washed with distilled water for $1 \mathrm{~h}$ at $4{ }^{\circ} \mathrm{C}$. Those roots were cut into $2 \mathrm{~mm}$ in length and treated with $4 \%$ Cellulase 'Onozuka' R10 (Yaklut Honsha) and 2\% Pectolyase Y-23 (Kikkoman) at pH 4.8, 37 ${ }^{\circ} \mathrm{C}$ for $1 \mathrm{~h}$. Then, they were washed with distilled water at $4^{\circ} \mathrm{C}$ for $1 \mathrm{~h}$, and chopped on a glass slide with $3: 1$ ethanol-acetic acid.

\section{Differential fluorescent staining with CMA and DAPI}

For fluorescent differential staining with CMA and DAPI, the method of Plader et al. (1998) was followed with slight modifications. The slides were pre-incubated in McIlvine's buffer ( $\mathrm{pH} 7.0)$ for $30 \mathrm{~min}$, and then treated with $0.1 \mathrm{mg} / \mathrm{ml} \mathrm{CMA}$ in McIlvine's buffer supplemented with $5 \mathrm{mM}$ $\mathrm{MgSO}_{4}$ for $10 \mathrm{~min}$. After rinsing, they were mounted in glycerol and stored at $4^{\circ} \mathrm{C}$ for at least $10 \mathrm{~h}$ to prevent fading. These chromosomes stained with CMA were irradiated with a BV filter cassette 

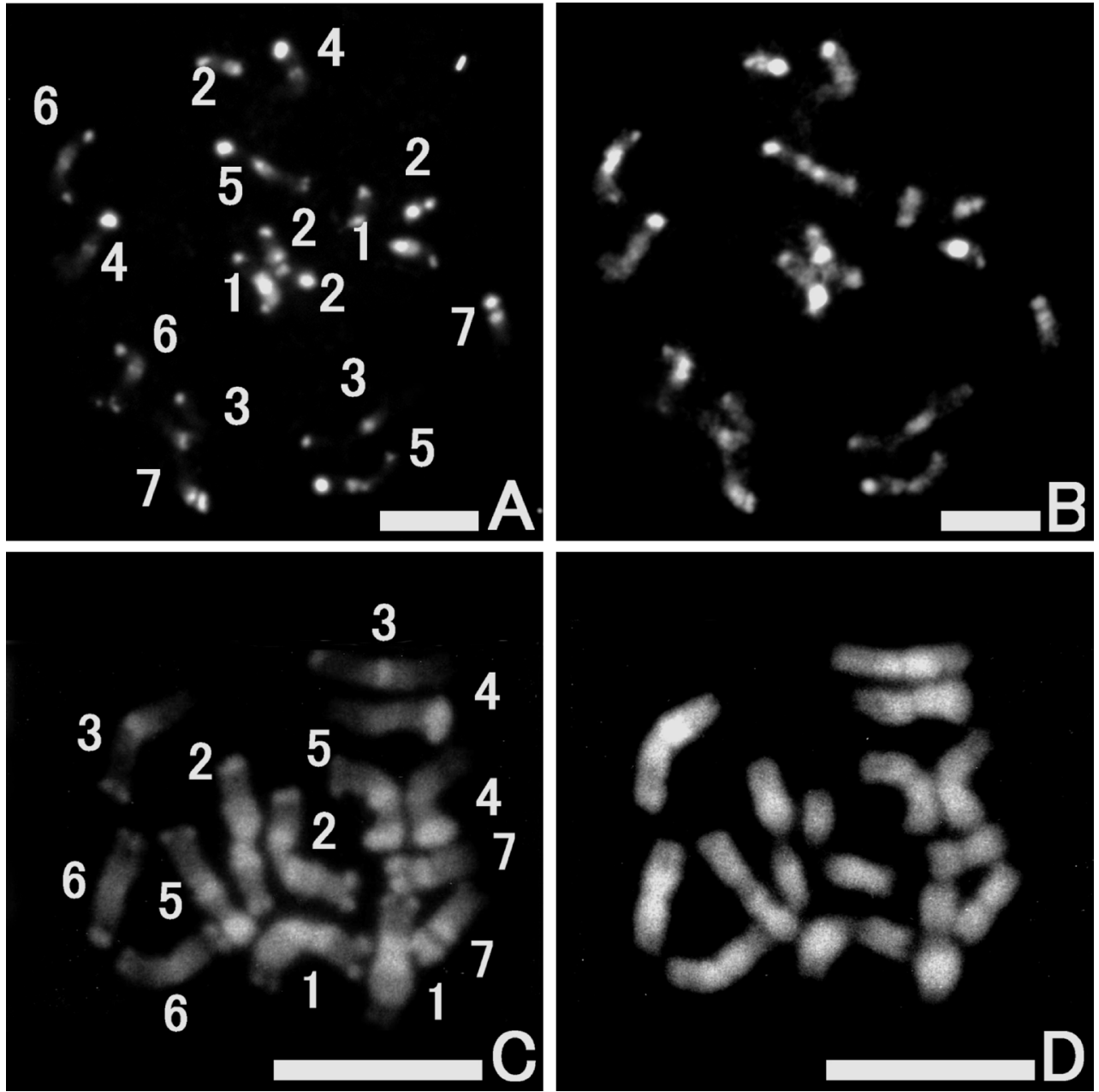

Fig. 1. Mitotic pro- (A, B) and early- (C, D) metaphase chromosome images of C. sativus cv. Borszczagowski stained with CMA (A, C) and DAPI (B, D). Identified chromosomes were indicated. Scale bar represents $5 \mu \mathrm{m}$ for A to D.

and fluoresced yellow. After CMA staining and observation, the slides were used for sequential DAPI staining. The slides were destained in $45 \%$ acetic acid for $30 \mathrm{~min}, 3: 1$ ethanol-acetic acid for $30 \mathrm{~min}$ and ethanol series $(30 \%, 70 \%$ and $99.5 \%(\mathrm{v} / \mathrm{v}))$ for $10 \mathrm{~min}$, respectively. They were dipped in McIlvine's buffer for $30 \mathrm{~min}$. And then, they were treated with $0.1 \mu \mathrm{g} / \mathrm{ml}$ DAPI in McIlvine's buffer for $10 \mathrm{~min}$. These chromosomes stained with DAPI were irradiated with a U filter cassette and fluoresced blue. Photographs were taken with SenSys (Photometrics) and Olympus fluorescent microscope (BX51). Chromosomes identification followed the procedure of Hoshi et al. (1999). Definition of prometaphase, early-metaphase and mid-metaphase stages in somatic cells followed Plader et al. (1998). Prometaphase chromosomes present clear banding pattern in CMA and DAPI staining. DAPI stained chromosomes do not show any clear band from early- to mid-metaphase, while the chromosomes stained with CMA show many bands. 

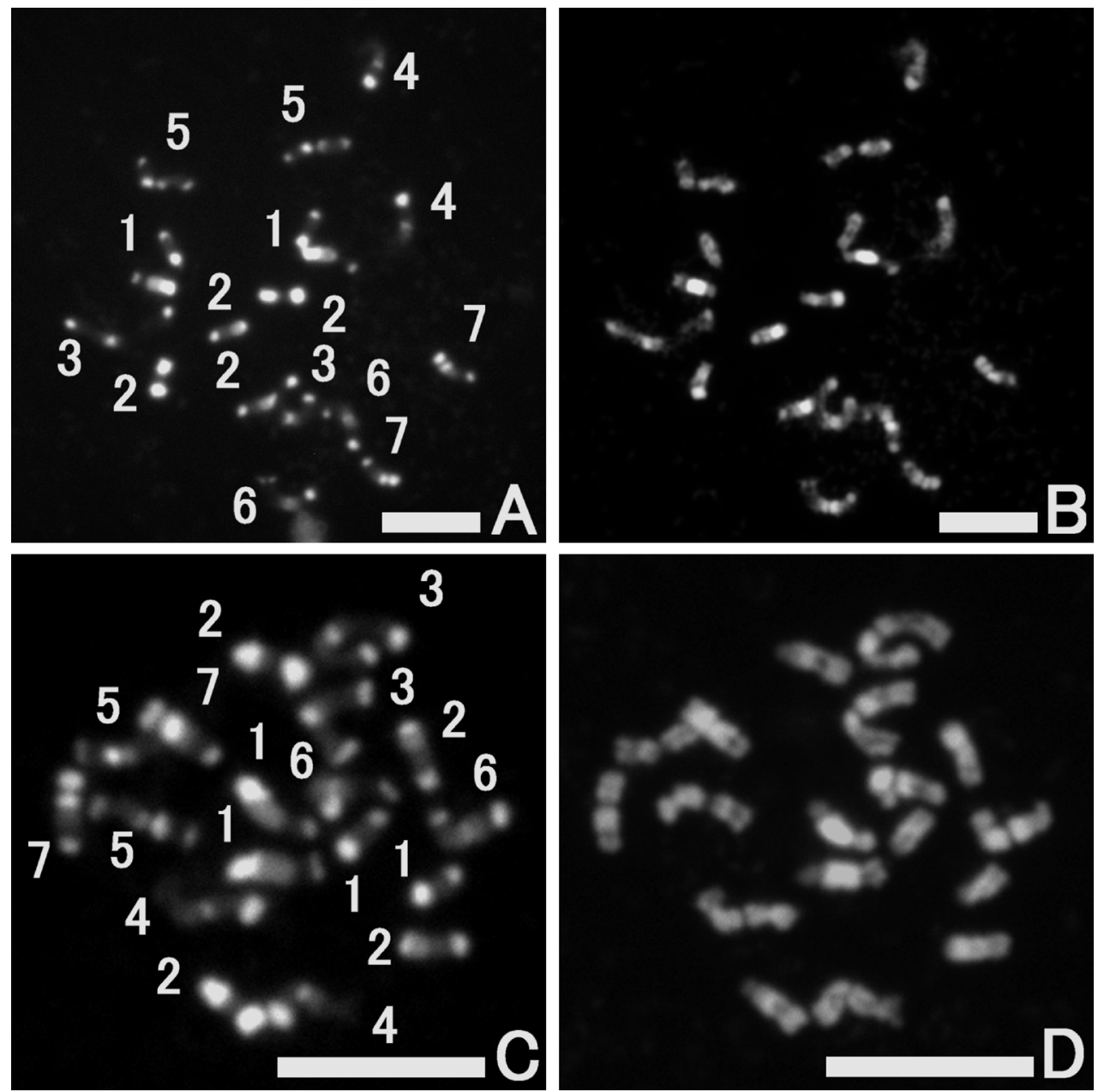

Fig. 2. Mitotic pro- (A, B) and early- $(\mathrm{C}, \mathrm{D})$ metaphase chromosome images of $C$. sativus cv. Monastyrski stained with CMA (A, C) and DAPI (B, D). Identified chromosomes were indicated. Scale bar represents $5 \mu \mathrm{m}$ for $\mathrm{A}$ to $\mathrm{D}$.

Result

Prometaphase and early-metaphase chromosomes were mainly analyzed for the comparative study. After the CMA and DAPI staining, most of the chromosomes displayed CMA-positive and DAPI-negative $\left(\mathrm{CMA}^{+} \mathrm{DAPI}^{-}\right)$, CMA-positive and DAPI-positive $\left(\mathrm{CMA}^{+} \mathrm{DAPI}^{+}\right)$, or CMA-negative and DAPI-positive $\left(\mathrm{CMA}^{-} \mathrm{DAPI}^{+}\right)$constitutive heterochromatic regions at pro-metaphase and early metaphase (Figs. 1, 2). These heterochromatic regions were mainly located on its terminal, interstitial and proximal regions of the chromosomes. With C. sativus cv. Borszczagowski chromosome complements as the basic $C$. sativus karyotype, prometaphase and early-metaphase chromosomes 1 to 7 of $C$. sativus cv. Monastyrski were compared and verificated (Figs. 3, 4).

Prometaphase chromosome 1 of $C$. sativus cv. Borszczagowski had the largest $\mathrm{DAPI}^{+}$segment from the proximal to middle part of the interstitial region of long arm, and $\mathrm{CMA}^{+} \mathrm{DAPI}^{-}$bands at the pericentromeric regions of both arms. The same banding pattern was observed in that of $C$. 
sativus cv. Monastyrski. In contrast, early-metaphase chromosome 1 did not possess clear $\mathrm{CMA}^{+} \mathrm{DAPI}^{+}$large segment. Well-defined elongated site at centromeric position of the chromosome 1 was often observed at prometaphase and early-metaphase cells of $C$. sativus cv. Borszczagowski and cv. Monastyrski.

Clear $\mathrm{CMA}^{+} \mathrm{DAPI}^{-}$segments were presented at the pericentromeric regions of chromosome 2 in $C$. sativus cv. Borszczagowski and cv. Monastyrski. In C. sativus cv. Borszczagowski chromosome 2, the banding patterns of long and short arms were similar. In contrast to C. sativus cv. Borszczagowski, long and short arms of chromosome 2 of $C$. sativus cv. Monastyrski could be easily distinguished, because large $\mathrm{CMA}^{+} \mathrm{DAPI}^{+}$heterochromatic band was observed at the terminal region of long arm, but not short arm.

Chromosome 3 had weakly $\mathrm{CMA}^{+} \mathrm{DAPI}^{-}$band at the pericentromeric region and $\mathrm{CMA}^{+} \mathrm{DAPI}^{+}$bands at the pericentromeric areas of both arms. Chromosome 3 in C. sativus cv. Monastyrski was characterized by $\mathrm{CMA}^{+} \mathrm{DAPI}^{+}$bands on the terminal regions of both arms, whereas that of $C$. sativus cv. Borszczagowski displayed only $\mathrm{CMA}^{+} \mathrm{DAPI}^{+}$band on the terminal region of long arm.

In chromosome 4, no difference was found in the banding patterns in both cultivars. The terminal region of short arm did not possess any heterochromatic bands, while the terminal region of long arm possessed large $\mathrm{CMA}^{+} \mathrm{DAPI}^{+}$heterochromatic band.

Chromosome 5 showed intraspecific polymorphism between two cultivars. Distinct $\mathrm{CMA}^{+} \mathrm{DAPI}^{+}$band located at terminal region of the long arm in C. sativus cv. Borszczagowski was larger than that of C. sativus cv. Monastyrski.

No heterchromatic band polymorphism was detected between chromosome 6 . Chromosome 6 had $\mathrm{CMA}^{+} \mathrm{DAPI}^{+}$bands at the pericentromeric and the terminal regions of short and long arms in both cultivars.

Chromosome 7, which was the smallest size in the chromosome complement, was characterized by evident $\mathrm{CMA}^{+} \mathrm{DAPI}^{+}$bands at the terminal regions of long arm, and $\mathrm{CMA}^{-} \mathrm{DAPI}^{+}$band located on the pericentromeric region of short arm in both of cultivars. Chromosomal polymorphism was recognized by terminal $\mathrm{CMA}^{+} \mathrm{DAPI}^{+}$band, which was only observed at short arm in $C$. sativus cv. Monastyrski.

\section{Discussion}

The earliest attempt of $C$. sativus chromosome identification was reported by Ramachandran and Seshadri (1986). They reported that karyotype formula was composed of two median, four submedian and one subterminal chromosomes when Japanese type cultivar was used as plant material. More detailed karyoptype analyses for chromosome identification were performed with C-banding techniques (Hoshi et al. 1998, Chen et al. 1998, Koo et al. 2002, 2005). Hoshi et al. (1998, 1999) and Plader et al. (1998) reported that the karyotype of European pickling type cultivar was composed of five metacentric and two submetacentric chromosomes by means of C-banding and fluorescent staining. In contrast, Chen et al. (1998) and Koo et al. (2002, 2005) reported that the haploid complement composed six metacentric and one submetacentric chromosome using North Chinese type and Korean type cultivars, respectively.

The karyotype of $C$. sativus cv. Borszczagowski in this study agreed with those of previous reports (Hoshi et al. 1998, 1999, Plader et al. 1998). However, our data was quite different from the karyotype reported by Chen et al. (1998) and Koo et al. (2002, 2005). Heterochromatin banding patterns of $C$. sativus cv. Borszczagowski chromosomes 1 and 4 were quite similar to those of chromosomes 4 and 6 in the report of Chen et al. (1998), and chromosomes 2 and 6 in the report of Koo et al. $(2002,2005)$, respectively. In contrast, the banding patterns of chromosomes 2, 3, 5 and 7 in the karyotype of C. sativus cv. Borszczagowski were not found in those of reported by Chen et al. 

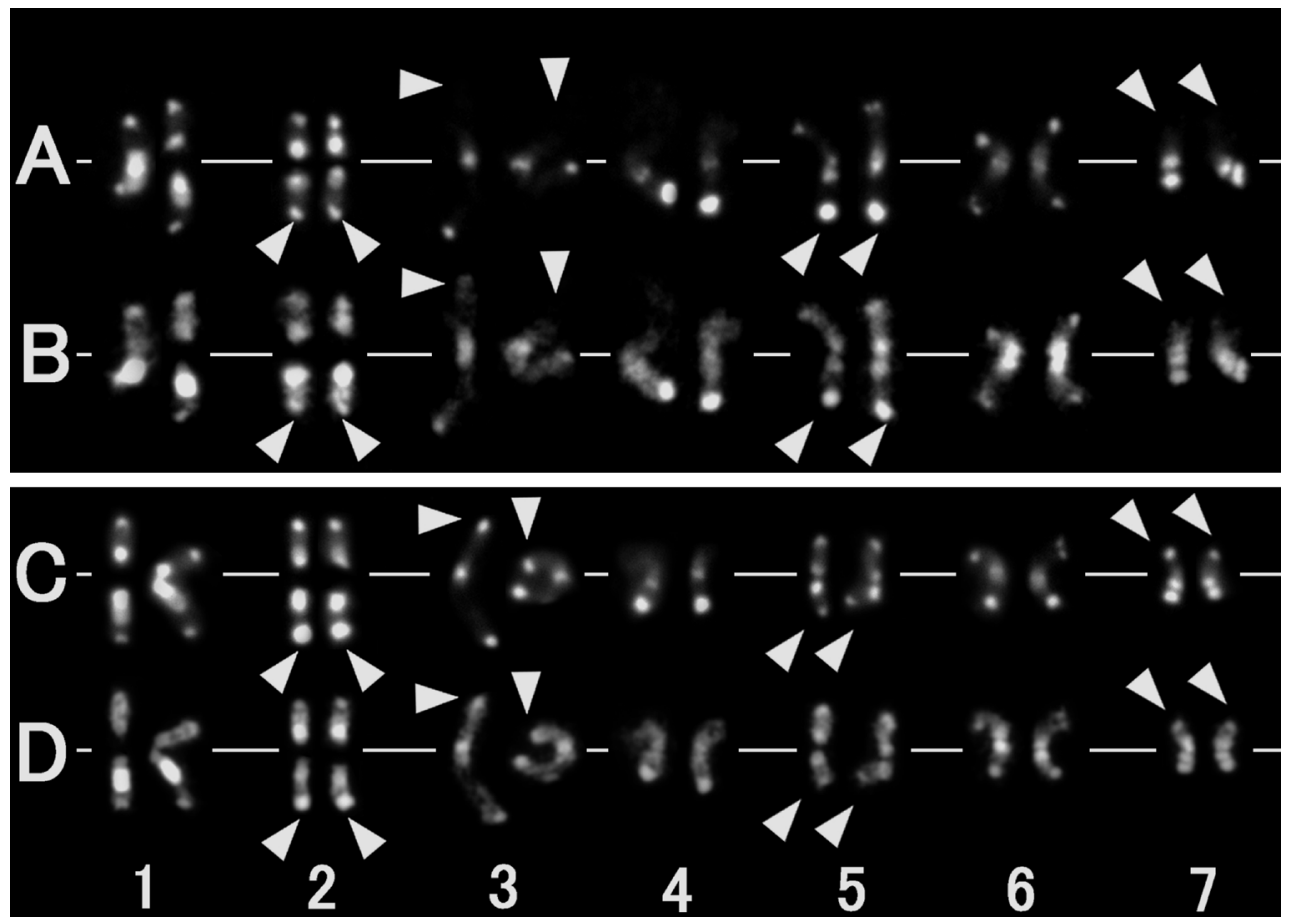

Fig. 3. Mitotic pro-metaphase karyotypes of $C$. sativus cv. Borszczagowski (A, B) and C. sativus cv. Monastyrski (C, D). Chromosomes were stained with CMA (A, C) and DAPI (B, D). Arrow heads were indicated chromosomal polymorphism sites between two cultivars.
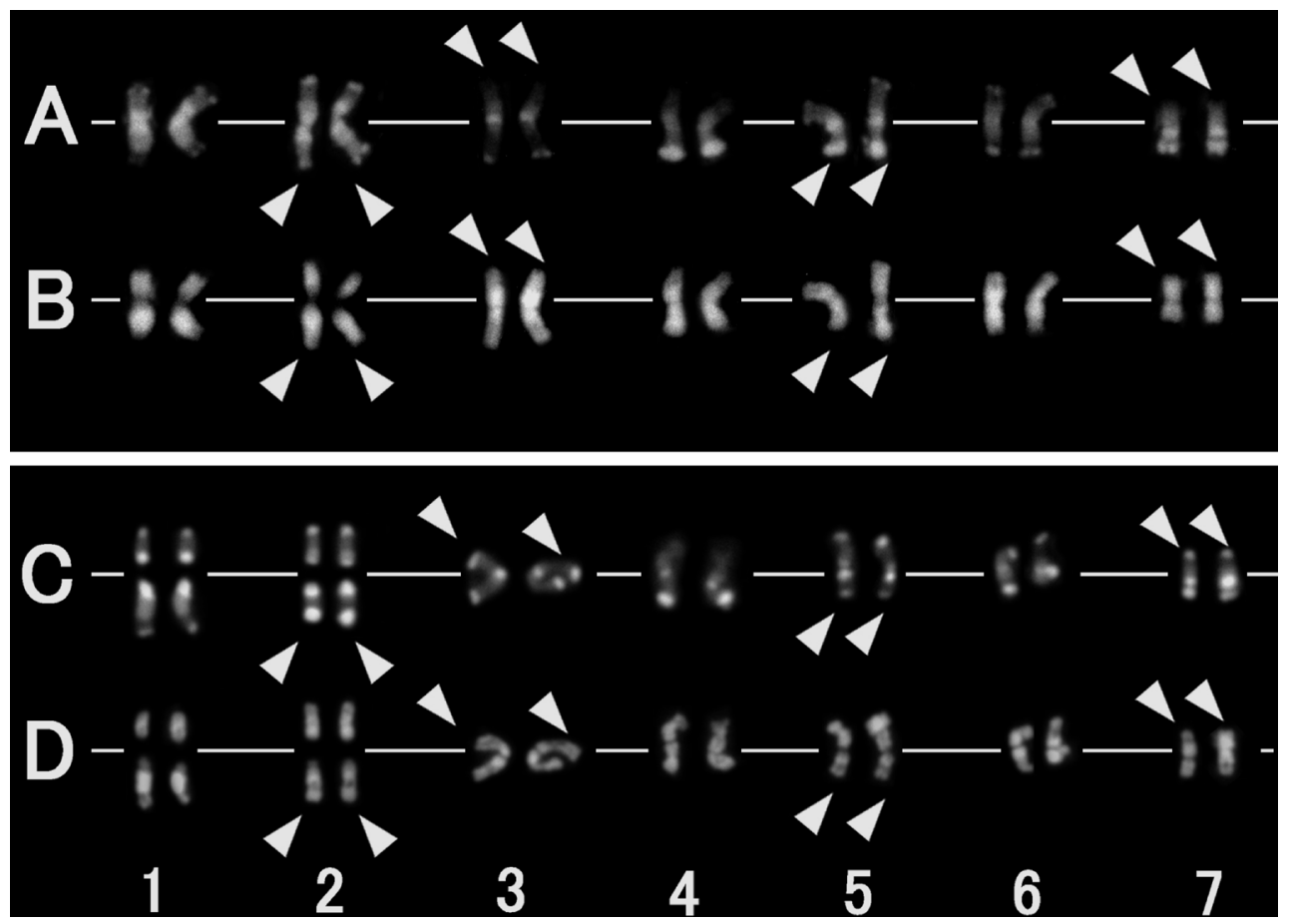

Fig. 4. Mitotic early-metaphase karyotypes of C. sativus cv. Borszczagowski (A, B) and C. sativus cv. Monastyrski (C, D). Chromosomes were stained with CMA (A, C) and DAPI (B, D). Arrow heads were indicated chromosomal polymorphism sites between two cultivars. 
(1998) and Koo et al. (2002, 2005). Our comparative result with two cultivars by using of the method for the same chromosome preparation showed that the cause of karyotype differences among cultivars is mainly due to intraspecific polymorphism.

Moreover, our study suggested that chromosome polymorphism in C. sativus was due to heterochromatin region structure. It is known that heterochromatic region-specific DNA is composed of highly repetitive sequences, such as Oryza sativa (Cheng et al. 2001) and Brassica rapa (Lim et al. 2005). These repetitive sequences are frequently deleted or duplicated, especially an event of unequal crossing-over during meiotic division (Smith 1976). Therefore, heterochromatic region polymorphisms in C. sativus might be related to this phenomenon.

The cultivated C. sativus is believed to be native to Western India (Candolle 1954), and includes pickling, South Chinese, North Chinese, Nepalese, and English types. There are following characteristic flute-shape in cultivation areas. Mature $C$. sativus flutes of pickling cultivars are rugby balled-shape with brown surface, while the flutes of South and North Chinese cultivars, which are most popular type in Japan, are elongated shape with green surface. Even thought quite large amounts of cultivars with different morphological characters are existed in the world, it is difficult to detect genetic variation among distantly related cultivars by linkage analysis of crossing experiments (Knerr et al. 1989). In this study, chromosome polymorphism was detected even in closely related cultivars of pickling cucumber. Thus, we expect that karyotypes among distantly related cultivars such as Japanese type may be more variable, and then chromosome identification will be more available to detect strain variations in C. sativus cultivar cultivated world wide. Further comparative chromosome studies between European and Asian cultivars will be necessary to clarify a whole picture of cucumber chromosome polymorphism.

\section{References}

Bentzer, B. and Landström, T. 1975. Polymorphism in chromosomes of Leopoldia comosa (Liliaceae) revealed by Giemsa staining. Hereditas 80: 219-232.

Candolle, D. E. (ed.) 1954. Origin of cultivated plants. Hafner pubishing. New York.

Chen, J. F., Sraub, J. E. and Jiang, J. 1998. A reevaluation of karyotype in cucumber (Cucumis sativus L.). Genet. Resour. Crop Evol. 45: 301-305.

Cheng, Z., Stupar, R. M., Gu, M. and Jiang, J. 2001. A tandemly repeated DNA sequence is associated with both knob-like heterochromatin and a highly decondensed structure in the meiotic pachytene chromosomes of rice. Chromosoma 110: $24-31$.

Hoshi, Y., Plader, W. and Malepszy, S. 1998. New C-banding pattern for chromosome identification in cucumber (Cucumis sativus L.). Plant Breeding 117: 77-82.

- , - and - 1999. Physical mapping of 45S rRNA gene loci in the cucumber using fluorescence in situ hybridization. Caryologia 52: 49-57.

Jeffrey, C. 1980. A review of the Cucurbitaceae. Bot. J. Linnean Soc. 81: 233-247.

Kakeda, K., Fukui, K. and Yamagata, H. 1991. Heterochromatic differentiation in barley chromosomes revealed by C- and N-banding techniques. Theor. Appl. Genet. 81: 144-150.

Knerr, L. D., Staub, J. E., Holder, D. J. and May, B. P. 1989. Genetic diversity in Cucumis sativus L. assessed by variation at 18 allozyme coding loci. Theor. Appl. Genet. 78: 119-128.

Koo, D. H., Hur, Y., Jin, D. and Bang, J. 2002. Karyotype analysis of Korean cucumber cultivar (Cucumis sativus L. cv. Winter Long) using C-banding and Bicolor fluorescence in situ hybridization. Mol. Cells. 13: 413-418.

—, Choi, H. W., Cho, J., Hur, Y. and Bang, J. W. 2005. A high-resolution karyotype of cucumber (Cucumis sativus L. 'Winter Long') revealed by C-banding, pachytene analysis, and RAPD-aided fluorescence in situ hybridization. Genome 48: 534-540.

Laursen, L. 1978. Giesa C-banding of barley chromosomes I. Banding pattern polymorphism. Hereditas 88: 55-64.

Lim, K. B., Jong, H., Yang, T. J., Park, J. Y., Kwon, S. J., Kim, J. S., Lim, M. H., Kim, J. A., Jin, M., Jin, Y. M., Kim, S. H., Lim, Y. P., Bang, J. W., Kim, H. I. and Park, B. S. 2005. Characterization of rDNAs and tandem repeats in the heterochromatin of Brassica rapa. Mol. Cells. 19: 436-444.

Plader, W., Hoshi, Y. and Malepszy, S. 1998. Sequential fluorescent staining with CMA and DAPI for somatic chromosome identification of cucumber (Cucumis sativus L.). J. Appl. Genet. 39: 249-258. 
Ramachandran, C. and Seshadri, V. S. 1986. Cytological analysis of the genome of cucumber (Cucumis sativus L.) and muskmelon (Cucumis melo L.) Z. Pflanzenzüchtg. 96: 25-38.

Schweizer, D. 1976. Reverse fluorescent chromosome banding with chromomycin and DAPI. Chromosoma 58: 307-324.

Smith, G. P. 1976. Evolution of repeated DNA sequences by unequal crossover. Science 191: 528-535.

Vosa, C. G. 1976. Chromosome banding patterns in cultivated and wild barleys (Hordeum spp). Heredity 37: 395-403.

- 1973a. Heterochromatin recognition and analysis of chromosome variation in Scilla sibirica. Chromosoma 43: $269-278$.

- 1973b. Quinacrine fluorescence analysis of chromosome variation in the plant Tulvaghia leucantha. Chromosome today 4: 345-349.

Weimarck, A. 1975. Heterochromatin polymorphism in the rye karyotype as detected by Girmsa C-banding technique. Heteritas 79: 293-300. 